\title{
Rational use of Computed Tomography Scan head in the Emergency Department of a high volume tertiary care public sector hospital
}

\author{
Tahira Nishtar ${ }^{1}$, Tabish Ahmad², \\ Nosheen Noor ${ }^{3}$, Fayaz Muhammad ${ }^{4}$
}

\begin{abstract}
Objective: To emphasize the rational use of Computed Tomography (CT) head in emergency department (ED) of a high volume tertiary care hospital.

Methods: This retrospective observational study was conducted in Radiology Department of Medical Teaching Institute Lady Reading Hospital (MTI-LRH), Peshawar, Pakistan from November $1^{\text {st }} 2017$ to $31^{\text {st }}$ January 2018. Patients of all ages and both genders presenting to the emergency department with post traumatic and non-traumatic indications for emergency CT head scan were included in the study. The imaging was performed on GE 16 multi slice Optima CT system. The imaging protocol included slice thickness of 3-5mm, non-contrast study for cases of head trauma or suspected stroke. Where needed intravenous contrast was administered e.g. to exclude meningitis in patients presenting with severe headache. Patients undergoing CT examination for regions of the body other than head and brain were excluded from the study as their number was insignificant. Reporting was done on PACS and results analyzed using latest SPSS version.

Results: Out of 4284 CT scans performed in emergency department $90.8 \%$ were CT head (3893). Among 3893 CT scan head done in ED, 2581 cases were reported normal (66.29\%), while 1312 cases had positive findings (33.7\%), including post traumatic and non-traumatic.

Conclusion: Misuse of CT head is common especially in an emergency setting. Emergency physicians should be encouraged to obtain a detailed history and perform a thorough physical examination with reference to internationally standardized guidelines, while ordering CT scan.
\end{abstract}

KEYWORDS: CT Scan, Emergency Department, Rational use.

doi: https://doi.org/10.12669/pjms.35.2.719

How to cite this:

Nishtar T, Ahmad T, Noor N, Muhammad F. Rational use of Computed Tomography Scan head in the Emergency Department of a high volume tertiary care public sector hospital. Pak J Med Sci. 2019;35(2):302-308. doi: https://doi.org/10.12669/pjms.35.2.719

This is an Open Access article distributed under the terms of the Creative Commons Attribution License (http://creativecommons.org/licenses/by/3.0), which permits unrestricted use, distribution, and reproduction in any medium, provided the original work is properly cited.

1. Dr. Tahira Nishtar, (FCPS),

Associate Professor and Chairperson,

2. Dr. Tabish Ahmad, PGR-FCPS II,

3. Dr. Nosheen Noor, (MCPS, FCPS),

Assistant Professor,

4. Dr. Fayaz Muhammad, PGR-FCPS II,

1-4: Radiology Department, Medical Teaching Institute, Lady Reading Hospital,

Peshawar, Pakistan.

Correspondence:

Dr. Nosheen Noor,

Assistant Professor,

Radiology Department,

MTI-Lady Reading Hospital,

Peshawar, Pakistan.

E-mail: noshyyn@hotmail.com

* Received for Publication:

* Revision Received:

* Revision Accepted:
September 26, 2018

January 18, 2019

January 29, 2019

\section{INTRODUCTION}

CT scan is an important investigating tool in the ED in establishing a particular diagnosis or otherwise helping a physician to exclude one. So it is not only used as a screening tool but also as a diagnostic one. ${ }^{1}$

The most common CT scan done in an emergency department is CT head, which falls under two categories i.e. post traumatic and non-traumatic CT head. ${ }^{2}$ There are risks related to overuse of CT scans as each scan involves radiation, not justifying scans done for marginal reasons. The overuse of CT scan is closely related to changing trends in medical practice and easy access to CT scan with lower barriers and thresholds to performing the test. ${ }^{3}$ 
A segment of CT scan in emergency department is done for medico-legal purposes requiring evidence based clinical practice. There are obvious benefits to judicious imaging in ED with less ionizing radiation exposure. Radiation from medical imaging causes long term cancer risk more significantly in children and young adults. In the elderly and high risk population with co-morbidities, repeated CT head does not identify acute clinically significant findings. ${ }^{4}$

There are certain pre-requisites for ordering a CT Scan of Head. It includes comprehensive history \& physical examination and documentation concerning relevant symptoms related to ordering a specific CT examination with reference to the standardized international guidelines for CT imaging in an emergency setting (Fig.1, 2 \& 3).

This is perhaps first study of its kind performed in a public sector hospital of the Pukhtunkhawa province of Pakistan. The purpose of this study was to emphasize on rational use of CT head in $\mathrm{ED}$ and to discourage misuse of CT scan in patient population.

\section{METHODS}

This retrospective observational study was conducted in Radiology Department of Medical Teaching Institute Lady Reading Hospital (MTILRH), Peshawar, Pakistan from November $1^{\text {st }} 2017$ to $31^{\text {st }}$ January 2018. Patients of all ages and both genders presenting to the emergency department with post traumatic and non-traumatic indications for CT head were included in the study. The imaging was performed on Optima 16 multi slice CT system (GE). The imaging protocol included slice thickness of $3-5 \mathrm{~mm}$, non-contrast study in case of trauma or stroke. Where needed intravenous contrast was administered e.g. to exclude meningitis. Patients undergoing CT examination for regions of the body other than head and brain were excluded from the study as their number was insignificant. In cases of stroke,
Table-I: CT Head (N=3893): Normal vs. Abnormal.

\begin{tabular}{|c|c|c|c|c|}
\hline \multirow[t]{2}{*}{ Indication } & \multicolumn{2}{|c|}{$\begin{array}{l}\text { Normal } \\
\text { CT head } \\
(2581)\end{array}$} & \multicolumn{2}{|c|}{$\begin{array}{l}\text { Abnormal } \\
\text { CT head } \\
\text { (1312) }\end{array}$} \\
\hline & & $\begin{array}{c}\text { Percentage } \\
\text { out of total } \\
N(\%)\end{array}$ & No. & $\begin{array}{c}\text { Percentage } \\
\text { out of total } \\
N(\%)\end{array}$ \\
\hline Non traumatic & 1330 & 34.16 & 778 & 19.98 \\
\hline Post traumatic & 1251 & 32.13 & 534 & 13.72 \\
\hline Total & 2581 & 66.29 & 1312 & 33.7 \\
\hline
\end{tabular}

finding of infarct rather than suspected bleed or vice versa were both considered as positive. Those patients who had normal CT were considered as negative. CT images were reported on PACS in two sessions of six hours each, with morning session reporting done by resident under the supervision of consultant radiologist while evening session reporting was performed by a senior Third or Fourth Year resident independently. Information was analyzed using latest SPSS version.

\section{RESULTS}

Out of 4284 patients, 3019 were male $(70.5 \%)$, while 1265 were female (29.5\%). 4284 CT scans included $90.8 \%$ CT head (3893). The rest of 9.13\% (391) cases included abdomino-pelvic, thoracic, and musculoskeletal. Among 3893 CT head scans done in ED, 2581 cases were reported normal, while 1312 cases had positive findings (Table-I). The cases with positive findings were further divided into two broad categories of indication; post-trauma and non-trauma cases (Table-II). Post traumatic cases included road traffic accidents (RTA), history of fall (HOF), fire arm injury (FAI) and physical assault, whereas non-trauma indications included stroke, unconsciousness, severe headache and clinically suspected meningitis or rarely to exclude space occupying lesion.

Table-II: CT Brain with positive findings $(\mathrm{N}=1312)$.

\begin{tabular}{lclc}
\hline \multicolumn{2}{c}{ Non trauma positive CT scans } & \multicolumn{2}{c}{ Post trauma positive CT scans } \\
\hline Intracranial hemorrhage & 232 & Subarachnoid hemorrhage & 43 \\
Cerebral infarcts & 309 & Subdural hemorrhage & 46 \\
Hydrocephalus & 26 & Extradural hemorrhage & 54 \\
Brain atrophy & 211 & Intraventricular hemorrhage & 26 \\
& & Skull bone fractures & 236 \\
& & Pneumocephalus & 129 \\
\hline Total & $778(59.3 \%)$ & Total & $534(40.7 \%)$ \\
\hline
\end{tabular}


Rational use of CT head in Emergency Department



Fig.1: NICE guidelines for selection of children for CT head scan. ${ }^{11}$

Pak J Med Sci $\quad$ March-April 2019 Vol. 35 No. 2 www.pjms.org.pk 


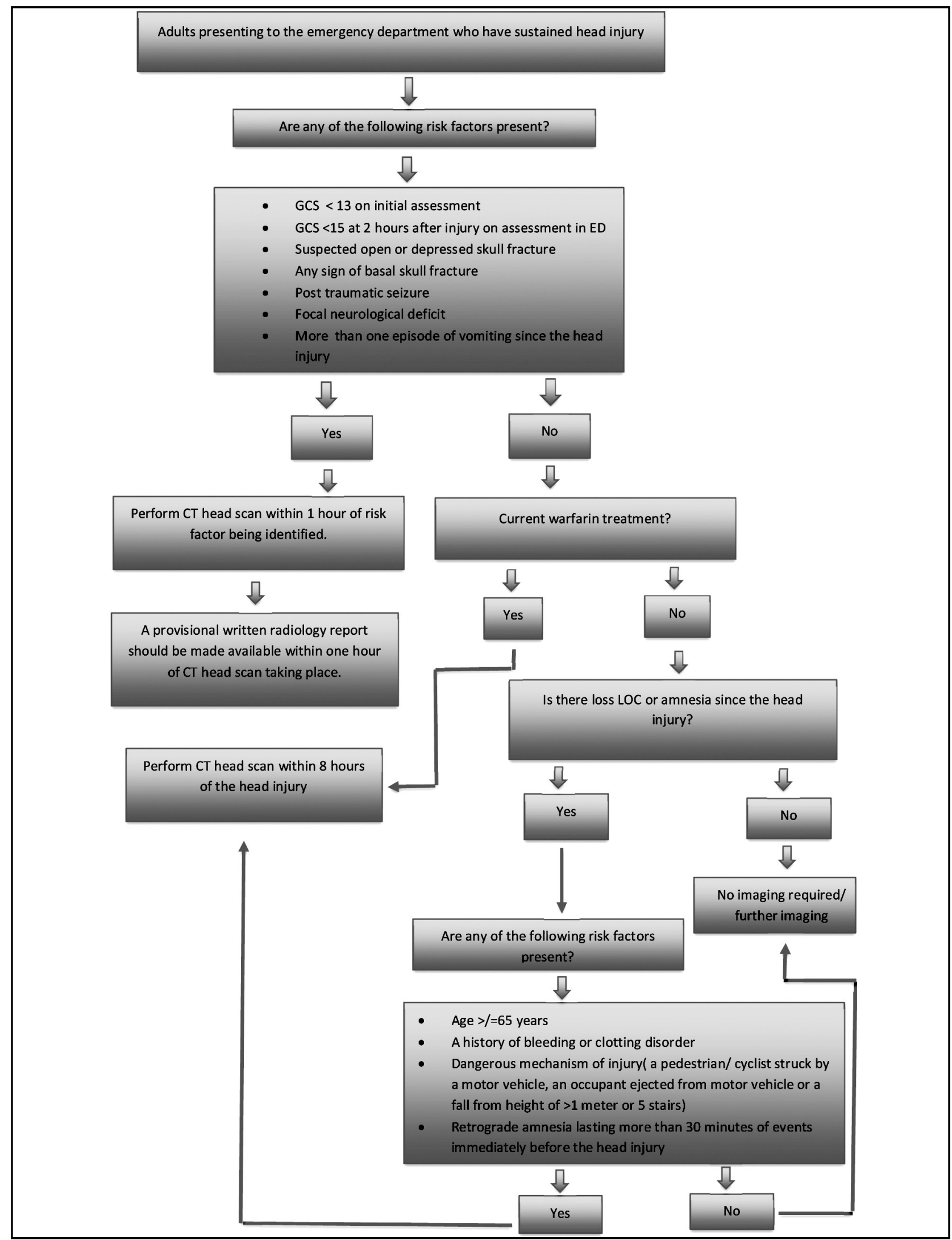

Fig.2. NICE guidelines for selection of adults for CT head scan. ${ }^{11}$ 


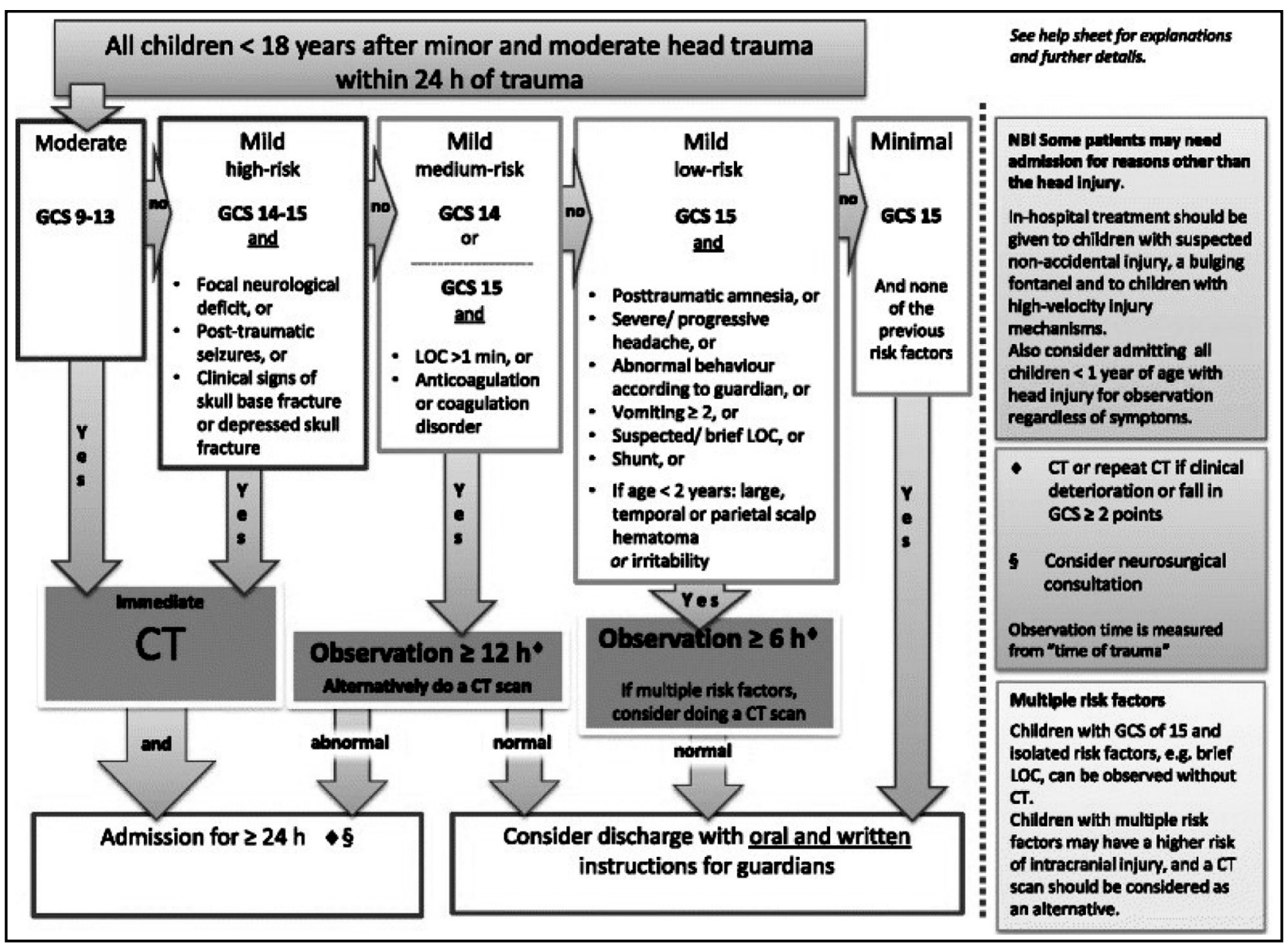

Fig.3: Scandinavian guidelines for initial management of minor and moderate head trauma in children. ${ }^{12}$

\section{DISCUSSION}

Access to free technical facilities in emergency department (ED) spares the patients from queuing up to seek specialist appointments. This results in overcrowding and increasing number of patients seeking emergency care for non-urgent cases leading to "misuse of emergency department services". The fact that emergency department offers a 24/7 free service adds to this overcrowding. Another factor contributing to emergency department overcrowding is in-adequate in-patient bed availability. Demand growth is mainly due to inaccessibility to primary healthcare. ${ }^{1}$

CT head is one of the most common scan prescribed by physicians in ED for various indications such as weakness, aphasia, headache, syncope, dizziness and trauma. However the number of CT scans ordered by the physicians varies from one hospital to another and from one physician to another because of lack of implementation of standardized protocols. The use of CT scans in the ED has significantly increased over the past decade. Some physicians rely more on their clinical history and examination findings while others on evidence of presence or absence of pathology in the form of a positive or negative CT scan. ${ }^{5}$ In this study conducted at ED, LRH, majority of the patients referred for CT head scan which were reported as normal presented with headache, history of seizures and minor trauma. In patients with minimal and mild head injury CT head was advised for screening. However, according to the international guidelines there is no need of undergoing an urgent CT head if GCS is 14 to 15 without risk factors, and either discharge or 6 to 12 hour observation is suggested accordingly, rather than performing screening CT head. The CT head scans with positive findings in our study were mainly post major trauma including RTA, falls, fire arm injuries and physical blow to the head. In cases of suspected stroke predominantly in elderly population, patients presenting with weakness, seizures, amnesia and loss of consciousness with or without focal neurological deficit underwent 




Fig.4: Scandinavian guidelines for initial management of minor and moderate head trauma in adults. ${ }^{13}$

CT head. The most common finding in elderly was senile brain atrophy which was included in positive scans.

It is very important to channelize the resources of hospital in an effective way in order to avoid not only unnecessary load on the ED CT services but also to decrease the radiation exposure to patients. Increased radiation exposure for patients visiting the ED has been a significant problem in recent years as the physicians working in ED have developed a low threshold for ordering such investigations. ${ }^{1}$ This trend has increased in the past few years. ${ }^{6}$ This increases the exposure of patients to unnecessary radiations; radiations that could have otherwise been avoided if the ED physician had taken a detailed history and performed a thorough physical examination with reference to the standardized guidelines before ordering a CT scan. ${ }^{3}$

The increased radiation exposure is associated with increased incidence of malignancies in the population. ${ }^{7}$ Children are particularly more sensitive to repeated radiation doses. ${ }^{8}$ On the other hand, elderly patients in the population are also at an increased risk particularly if there is gross abuse of emergency CT scan services. ${ }^{7,8}$

Over-ordering of unnecessary CT scans increases the duration of stay in ER which not only wastes the precious time of the health care providers working in the ED but also increases the exposure of such patients to various hospital acquired infections, which in most cases are drug resistant and hard to treat, thus increasing the overall morbidity and mortality in the population. ${ }^{9}$ Additionally it leads to overcrowding of the ED causing significant problems in delivery of quality services to the more deserving patients. ${ }^{1}$ One important reason for the overuse of CT services in the ED is the increased pressure on the ED physicians to make rapid and precise diagnosis in the shortest possible time. The pressure under which the ER physicians have to work forces them to order CT scans for increasing number of patients in order to screen them against common pathologies presenting to $\mathrm{ED}$ without taking a detailed history or performing a thorough physical examination. ${ }^{2}$ This indiscriminate use of 
CT scans in the ED has led to an enormous amount of financial burden on the hospital resources. ${ }^{6}$ The misuse of financial resources could otherwise prove very helpful if properly channelized to improve other services provided by the emergency department. ${ }^{9}$

In our study all the CT head scans done in the emergency department during the study period were included. The results showed that CT head was the most commonly prescribed CT scan in the ED. Among these $66.29 \%$ of scans were normal while $33.7 \%$ scan had positive findings. Thus almost two third of the total CT head scans done in the emergency department were normal. This shows a misuse of the emergency CT services. The reasons being high volume of patients, inadequate staffing, time constraints leading to lack of detailed history taking and physical examination, availability of free CT services, uncertainty on clinical diagnosis in most instances on part of some ED physicians; important factor being lack of awareness/ implementation of internationally standardized guidelines as a reference before ordering CT scan. ${ }^{10}$

As a result of this study radiology department LRH devised and implemented radiology request forms for quality assurance for selection of both adults and children for CT scan head presenting to $\mathrm{ED}$ in order to ensure judicious and rational use of $\mathrm{CT}$ services in an emergency setting.

\section{CONCLUSION}

CT head overuse is common especially in an emergency setting. Emergency physicians should be encouraged to perform a detailed history \& physical examination and exercise overall judicious rational use of CT scan especially in pediatric CT head keeping the international guidelines as a standard protocol for patient selection.

\section{Grant Support \& Financial Disclosures: None.}

\section{REFERENCES}

1. Li CJ, Syue YJ, Lin YR, Cheng HH, Cheng FJ, Tsai TC, Chen KF, Lee $\mathrm{CH}$. Influence of $\mathrm{CT}$ utilization on patient flow in the emergency department: a retrospective 1-year cohort study. BMJ Open. 2016;6(5):e010815. doi: 10.1136/ bmjopen-2015-010815.

2. Bellolio MF, Heien HC, Sangaralingham LR, Jeffery MM, Campbell RL, Cabrera D, Shah ND, Hess EP. Increased Computed Tomography Utilization in the Emergency Department and its Associationwith Hospital Admission. West J Emerg Med. 2017;18(5):835-845. doi: 10.5811/ westjem.2017.5.34152.
3. Parente DB. Radiation risk in the indiscriminate use of CT scan. Radiologia Brasileira. 2013;46(2):v-vi. doi 10.1590/ S0100-39842013000200001.

4. Ip IK, Schneider L, Seltzer S, Smith A, Dudley J, Menard A, Khorasani R. Impact of provider-led, technology-enabled radiology management program on imaging. Am J Med. 2013;126(8):687-692. doi: 10.1016/j.amjmed.2012.11.034.

5. Dolan TG. CT in the ER - Radiologists and Emergency Physicians Often See Radiation Risks and Benefits Differently. Radiol Today. 2011;12(7):16.

6. Larson DB, Johnson LW, Schnell BM, Salisbury SR, Forman HP. National trends in CT use in the emergency department: 1995-2007. Radiology. 2011;258(1):164-173. doi: 10.1148/ radiol.10100640.

7. Berrington de Gonzalez A, Mahesh M, Kim KP, Bhargavan M, Lewis R, Mettler F, Land C. Projected Cancer Risks From Computed Tomographic Scans Performed in the United States in 2007. Arch Intern Med. 2009;169(22):2071-2077. doi:10.1001/archinternmed.2009.440.

8. Brenner DJ, Elliston CD, Hall EJ, Berdon WE. Estimates of the cancer risks from pediatric $\mathrm{CT}$ radiation are not merely theoretical: Comment on "point/counterpoint: in x-ray computed tomography, technique factors should be selected appropriate to patient size against the proposition". Med Phys. 2001;28: 2387-2388.

9. Saber Tehrani AS, Diarmuid C, Hsieh YH, Mantokoudis G, Korley FK, Kerber KA, Frick KD, Newman-Toker DE. Rising Annual Costs of Dizziness Presentations to U.S. Emergency Departments. Acad Emerg Med. 2013;20:689696. doi: 10.1111/acem.12168.

10. Sierzenski PR, Linton OW, Amis ES Jr, Courtney DM, Larson PA, Mahesh M, et al. Applications of justification and optimization in medical imaging: examples of clinical guidance for computed tomography use in emergency medicine. Ann Emerg Med. 2014;63(1):25-32. doi: 10.1016/j. annemergmed.2013.08.027

11. Tessa Davis, Anna Ings. Head injury: triage, assessment, investigation and early management of head injury in children, young people and adults (NICE guideline CG 176). BMJ ADC Educ Pract. 2014;100:2. doi:10.1136/ archdischild-2014-306797

12. Ramona Astrand, Christina, Rosenlund, Johan Unden. Scandinavian guidelines for initial management of minor and moderate head trauma in children. BMC Med. 2016;14:33. doi: 10.1186/s12916-061-0574-x

13. Johan Uden, Tor Ingebrigtsen, Bertil Romner and the Scandinavian Neurotrauma Committee. Scandinavian guidelines for initial management of minimal, mild and moderate head injuries in adults: An evidence and consensus-based update. BMC Med. 2013;11:50. doi: 10.1186/1741-7015-11-50

\section{Author`s Contribution:}

TN conceived and final approval of article.

TN, TA and NN did manuscript writing.

TA, NN and FM did data collection.

TA and FM prepared the results.

NN did manuscript editing, review and final approval of manuscript. 